It thus appears that two types of spectra have been visible from March 19 to May 3. Photographs of the peculiar spectrum were obtained on March 19, April 12, 26, 28, May I, and 3 ; of the normal spectrum on March $23,27,30$, April I, 13, and 27. It is interesting to note the connection between the changes in the spectrum and in the light of the Nova, for on all the dates on which the peculiar spectrum was photographed, a minimum occurred, according to the Harvard visual and photometric observations, except on April 12. The magnitude of the star was the same on April 12 and I 3 , while the spectrum was different. On April 26 and 28, however, there occurred marked minima, and the peculiar spectrum was photographed on both dates, while on the intermediate evening, April 27, when the Nova was about a magnitude and a half brighter than on either the preceding or following night, the spectrum was normal.

The following table shows the connection between the sprectrum and the magnitude of the Nova. The first column gives all the dates on which the Nova was successfully photographed at Harvard with the $1 \mathrm{I}$ inch telescope from March 17 to May 3, I 901 . The second column gives the character of the spectrum. The third column gives the magnitude from visual observations reduced to the photo. metric scale.

Relation of Spectrum to Magnitude.

\begin{tabular}{|c|c|c|c|c|c|c|c|c|}
\hline 1901 & Spectrum & Magn & 1901 & Spectrum & Magn. & I 901 & Spectrum & Magn. \\
\hline Mar. 17 & Normal & 3.8 & April I & Normal & 4.1 & April 27 & Normal & 4.2 \\
\hline 19 & Peculiar & 50 & 12 & Peculiar & 4.6 & 28 & Peculiar & $5 \cdot 4$ \\
\hline 23 & Normal & 3.6 & 13 & Normal & 4.6 & May I & Peculiar & $5 \cdot 3$ \\
\hline 27 & Normal & 4. I & 26 & Peculiar & 5.8 & 3 & Peculiar & $5 \cdot 5$ \\
\hline 30 & Normal & 4.2 & & & & & & \\
\hline
\end{tabular}

The Spectrum of $\eta$ Carinae ( $\eta$ Argus).

On April 16, 1898, Miss Cannon, while examining plates taken in Arequipa for the classification of stellar spectra, recorded the statement regarding Nova Aurigae, -Spectrum made up almost entirely of bright bands that coincide in position with those of $\eta$ Carinae. This is followed by a detailed description of the spectrum of $\eta$ Carinae, and a comparison with the spectrum of Nova Aurigae, in.

Harvard College Observatory, 190 I June 6. cluding the bright and dark hydrogen lines, which is incorporated in the Remarks in the Annals, Vol. XXVII, page I 75, now in the hands of the printer. Photographs pointing out this resemblance were contained in the exhibit of the Harvard Observatory at Paris in 1900, and are now in the Buffalo Exhibition.

\title{
Observations of Planets at Windsor, N. S. Wales.
}

The observations were all made with a filar micrometer on the 8 inch equatorial in a bright field, and have been corrected for micrometer error and refraction. The transits of both limbs of Jupiter were observed in each comparison and the north and south limbs were observed alternately.
Mr. Merfield of Sydney has kindly communicated the resulting corrections to the ephemerides. Here Michajlovski's ephemeris of (78) Diana was not received till four days after the opposition and cloudy weather prevented observation after February 25.

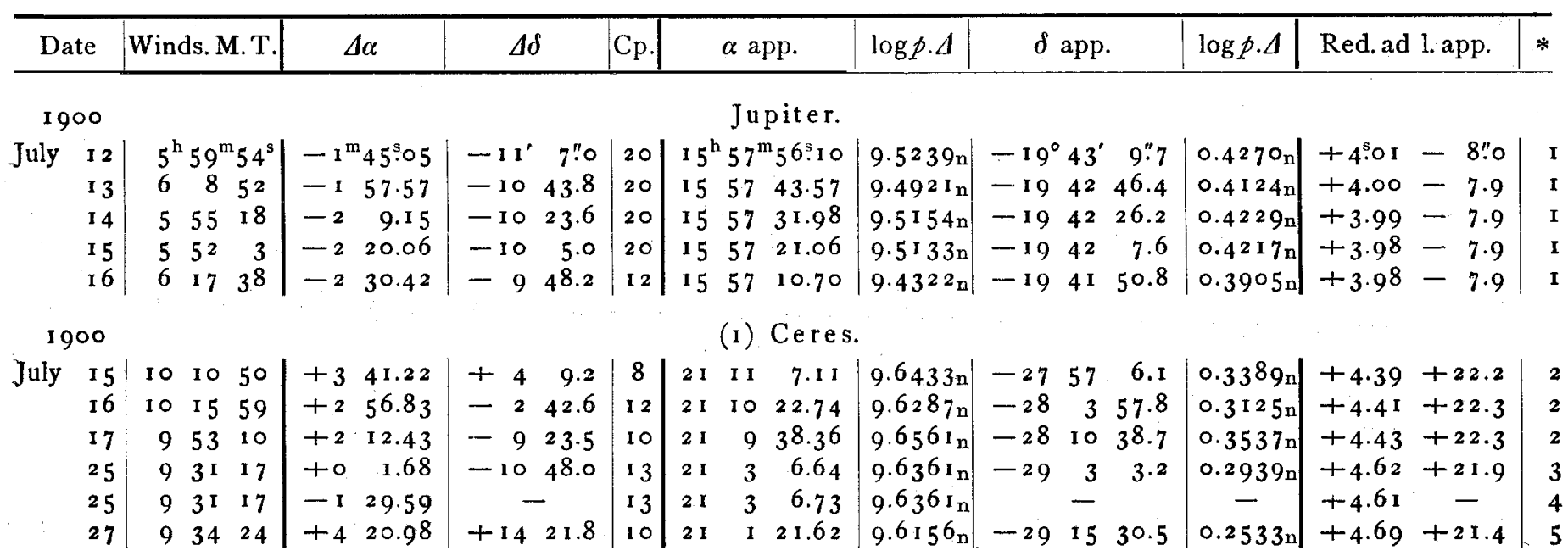






1900

\begin{tabular}{rr|rrr|rr|rr|r|} 
Sept. 18 & 10 & 4 & 9 & +3 & 49.27 & -17 & 33.7 & 10 \\
& 27 & 8 & 42 & 13 & +2 & 9.52 & -4 & 40.4 & 14 \\
Oct. & 30 & 8 & 27 & 37 & +0 & 20.19 & -7 & 22.3 & 20 \\
& 1 & 9 & 55 & 49 & -0 & 55.84 & +7 & 37.5 & 10 \\
& 2 & 9 & 1 & 9 & -1 & 34.15 & -5 & 13.6 & 10 \\
6 & 9 & 51 & 41 & -1 & 17.07 & + & 4 & 55.2 & 10 \\
& 7 & 8 & 34 & 0 & -1 & 53.64 & -7 & 6.0 & 12 \\
8 & 8 & 42 & 4 & +2 & 19.07 & -8 & 20.7 & 10
\end{tabular}

(3) Juno.

- 32 I $5.81\left|9.5008_{n}\right|-12640.4|0.6745 n|+4.38+28.3 \mid 11$ \begin{tabular}{lll|l|l|l|l|l|l|}
0 & 26 & 45.77 & $9.5792_{n}$ & -32742.2 & $0.6606 n$ & $2.44+28.7$ & 12
\end{tabular} \begin{tabular}{lll|lll|ll|l}
$\circ$ & 24 & 47.34 & $9.5809 n$ & -4 & 8 & 16.7 & $0.6552 n+4.46+28.6$ & 13
\end{tabular}

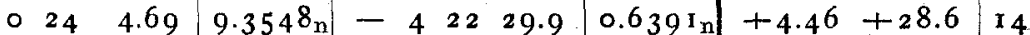
$02326.399 .5001_{n}-43521.00 .6438 n+4.47+28.6 \times 4$ $0.204^{6.71} 9.2785 n-52758.6 \quad 0.6237 n+4.48+28.5$ I5 $02010.14 \mid 9.5103 n-53959.80 .633^{1} n+4.48+28.5$ 1 5 ○I9 $31.66\left|9.4812_{n}-55238.4\right| 0.6281_{n} \mid+4.48+28.5$ I 6

(6) Hebe.

June $22 \mid$\begin{tabular}{lll|ll|}
10 & 31 & 13 & +3 & 26.04
\end{tabular} \begin{tabular}{l|lll|ll}
24 & 9 & 13 & 28 & +1 & $33 \cdot 7$ \\
\hline
\end{tabular}



\begin{tabular}{l|lll|ll}
26 & 8 & 52 & 44 & -0 & 22.94
\end{tabular}

\begin{tabular}{llll|ll}
27 & 9 & 10 & 2 & -1 & 23.09
\end{tabular}

$\begin{array}{lllllll}28 & 8 & 59 & 42 & -2 & 22.27\end{array}$

\begin{tabular}{r|rrr|rr}
30 & 9 & 42 & 33 & -4 & 23.96 \\
1 & 8 & 48 & 40 & -5 & 21.60
\end{tabular}

July

$9497+316.64$

9 \begin{tabular}{lll|l}
9 & 25 & +2 & 18.66
\end{tabular}

$\begin{array}{lllll}8 & 44 & 19 & -3 & 53.73\end{array}$

\begin{tabular}{lll|ll}
8 & 32 & 34 & -4 & 49.23
\end{tabular}

\begin{tabular}{rrr|rr}
8 & 30 & 54 & -5 & 44.20 \\
8 & 39 & 8 & -5 & 25.25
\end{tabular}

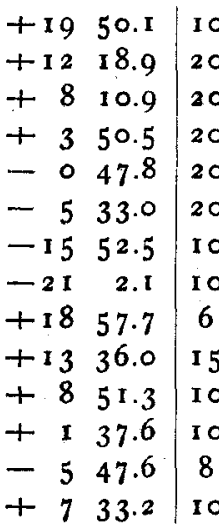

I 901

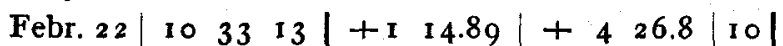
$23102147 \mid$ to $13.63+332.915$

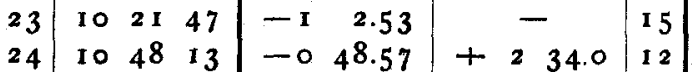

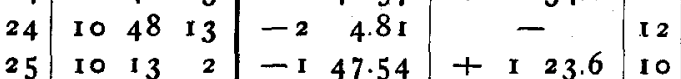

$25 \mid$\begin{tabular}{lll|ll|l|}
10 & 13 & 2 & -3 & 3.68 & +324.2
\end{tabular}

\begin{tabular}{|rrr|r|rrr|r|r|r}
18 & 31 & 31.43 & $9.3910_{n}$ & -5 & 39 & 11.3 & $0.6251 n$ & $+3.92+5.8$ & 17 \\
18 & 29 & 39.14 & $9.5575 n$ & -5 & 46 & 42.3 & $0.6373 n$ & $+3.94+6.0$ & 17 \\
18 & 28 & 40.97 & $9.5635 n$ & -5 & 50 & 50.1 & 0.6376 & $+3.96+6.2$ & 17 \\
18 & 27 & 42.50 & $9.5761 n$ & -5 & 55 & 10.4 & $0.6387 n$ & $+3.97+6.3$ & 17 \\
18 & 26 & 42.36 & $9.5365 n$ & -5 & 59 & 48.6 & $0.6325 n$ & $+3.98+6.4$ & 17 \\
18 & 25 & 43.19 & $9.5470_{n}$ & -6 & 4 & 33.7 & $0.6329 n$ & $+3.99+6.5$ & 17 \\
18 & 23 & 41.53 & $9.4211_{n}$ & -6 & 14 & 52.9 & $0.6194 n$ & $+4.02+6.8$ & 17 \\
18 & 22 & 43.90 & $9.5400_{n}$ & -6 & 20 & 2.4 & $0.6295 n+4.03+6.9$ & 17 \\
18 & 22 & 41.29 & $9.3844 n$ & -6 & 20 & 17.5 & $0.6161 n$ & $+4.04+6.2$ & 18 \\
18 & 21 & 43.32 & $9.5054 n$ & -6 & 25 & 39.1 & $0.6243 n$ & $+4.05+6.3$ & 18 \\
18 & 12 & 56.26 & $9.4347 n$ & -7 & 23 & 45.6 & $0.6060_{n}$ & $+4.13+6.9$ & 19 \\
18 & 12 & 0.77 & $9.4542 n$ & -7 & 30 & 59.2 & $0.6062 n$ & $+4.14+7.0$ & 19 \\
18 & 11 & 5.80 & $9.4455 n$ & -7 & 38 & 24.3 & $0.6040_{n}$ & $+4.14+7.1$ & 19 \\
18 & 9 & 17.66 & $9.3912 n$ & -7 & 53 & 42.6 & $0.5961 n$ & $+4.15+7.0$ & 20
\end{tabular}

(78) Diana.

$95936.63|9.2355 n|+132540.3|0.8027 n|+2.73-17.2 \mid 21$ 9 \begin{tabular}{lll|l|l|l|l|l|l|}
98 & 35.37 & $9.2679 n$ & +13 & 24 & 46.4 & $0.8015 n+2.73-17.2$ & 21
\end{tabular} $95835.459 .2679 \mathrm{n}-2 \mathrm{n}-2.73-2$

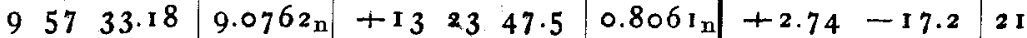

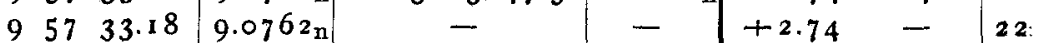
$95634.2109 .2627 \mathrm{n}+132247.1 \quad 0.8015 \mathrm{n}+2.74-17.221$ $95634.31|9.2627 n|+132246.8|0.8015 n|+2.74-17.3 \mid 22$ 
Mean Places of the Comparison Stars for the Beginning of the Year of Observation.

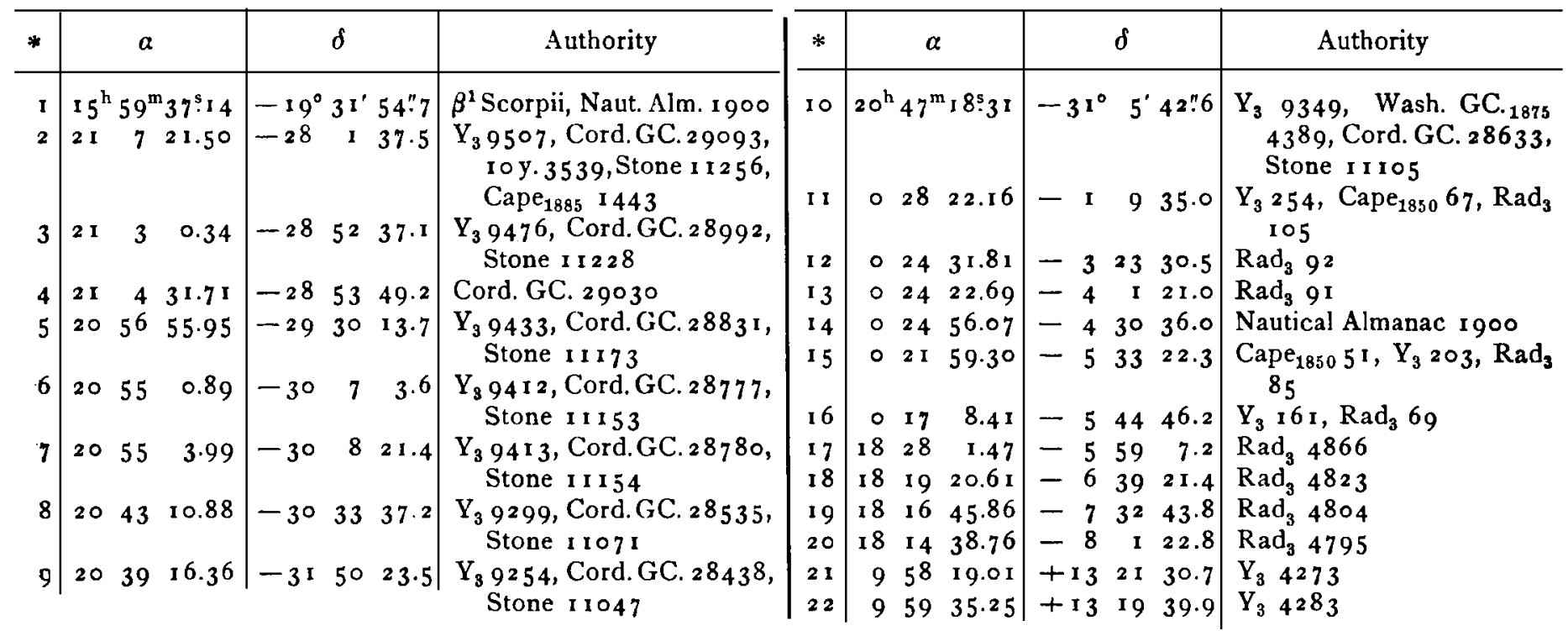

Comparison of observations with ephemerides $(\mathrm{O}-\mathrm{C})$.

\begin{tabular}{c|c|c}
\hline Date & $\Delta \alpha$ & $\Delta \delta$ \\
\hline & Jupiter. &
\end{tabular}

[N. A. 1900. Noon Ephemeris].

\begin{tabular}{rr|l|l}
\multicolumn{2}{c|}{ I900 } & & \\
& I 2 & +0.22 & -0.4 \\
1 3 & +0.18 & +0.2 \\
1 4 & +0.16 & +0.1 \\
1 5 & +0.19 & +0.4 \\
1 6 & +0.25 & +0.8
\end{tabular}

(1) Ceres.

[N. A. I 900. Transit Ephemeris].

\begin{tabular}{rr|c|c}
\multicolumn{2}{c|}{ I900 } & \\
July 15 & +1.87 & +5.6 \\
16 & +1.77 & +4.5 \\
17 & +1.80 & +4.2 \\
25 & +1.73 & +7.2 \\
25 & +1.82 & - \\
27 & +1.91 & +4.5 \\
28 & +2.06 & +4.5 \\
29 & +2.00 & +3.8 \\
Aug. & +1.97 & +4.5 \\
4 & +2.03 & +3.7 \\
4 & +1.98 & - \\
4 & +1.93 & +4.3 \\
5 & +1.92 & -
\end{tabular}

\begin{tabular}{rr|c|c}
\hline \multicolumn{1}{c|}{ Date } & $\Delta \alpha$ & $\Delta \delta$ \\
\hline Aug. & 6 & +158. & +4.0 \\
& 6 & +1.87 & - \\
& 9 & +1.78 & +4.3 \\
& 10 & +1.89 & +4.4 \\
11 & +1.93 & +3.5 \\
12 & +1.86 & +4.2 \\
113 & +1.96 & +4.2 \\
14 & +1.90 & +3.8 \\
15 & +1.97 & +4.0 \\
17 & +1.98 & +3.0 \\
18 & +2.00 & +2.8 \\
19 & +1.93 & +2.6 \\
19 & +1.98 & +2.3 \\
21 & +1.78 & +2.9 \\
21 & +1.78
\end{tabular}

(3) Juno.

[N. A. 1900. Transit Ephemeris].

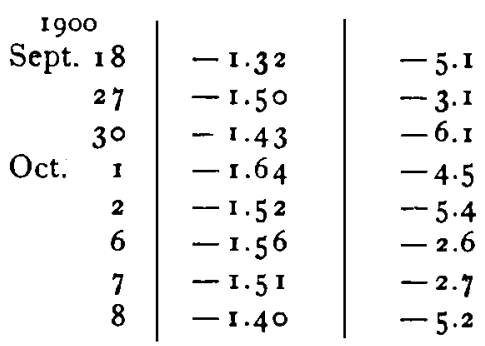

\begin{tabular}{|c|c|c|c|c|}
\hline \multicolumn{2}{|c|}{ Date } & $\Delta \alpha$ & & $\Delta \delta$ \\
\hline \multicolumn{5}{|c|}{ (6) Hebe. } \\
\hline \multirow[t]{7}{*}{ June } & 22 & $+4^{5.22}$ & - & 20.9 \\
\hline & 24 & +4.25 & - & 2 r.6 \\
\hline & 25 & +4.28 & - & 21.0 \\
\hline & 26 & +4.29 & - & $2 \mathrm{I} \cdot 3$ \\
\hline & 27 & +4.23 & - & 21.7 \\
\hline & 28 & +4.25 & - & 22.1 \\
\hline & $3^{\circ}$ & +4.27 & - & $22 \cdot 3$ \\
\hline \multirow[t]{7}{*}{ July } & I & +4.40 & - & 21.4 \\
\hline & I & $+4.3^{8}$ & - & 22.7 \\
\hline & 2 & +4.35 & - & $2 \mathrm{I} .4$ \\
\hline & II & +4.22 & - & $21 \cdot 3$ \\
\hline & 12 & +4.17 & - & 21.6 \\
\hline & 13 & +4.27 & - & 21.2 \\
\hline & 15 & +4.09 & - & 199 \\
\hline
\end{tabular}

(78) Diana.

[Michajlovski's Ephemeris].

\begin{tabular}{r|c|c}
\multicolumn{1}{c|}{ I901 } & \\
Febb.22 & +28.50 & -267.9 \\
23 & +28.36 & -266.8 \\
23 & +28.44 & - \\
24 & +28.51 & -265.5 \\
24 & +28.51 & - \\
25 & +28.32 & -264.1 \\
25 & +28.42 & -264.4
\end{tabular}

Fohn Tebbutt.

Planet (311) Clandia. Vergeblich gesucht rgor Juni rg und 22. M. Wolf. 